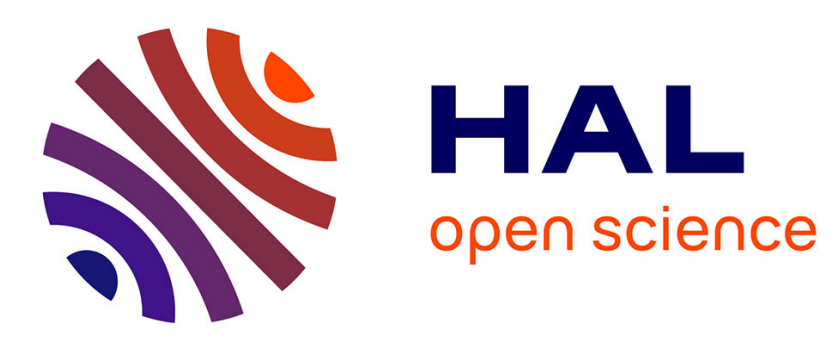

\title{
Coupling of a two phase gas liquid compositional 3D Darcy flow with a 1D compositional free gas flow
}

\author{
Konstantin Brenner, Roland Masson, L Trenty, Yumeng Zhang
}

\section{To cite this version:}

Konstantin Brenner, Roland Masson, L Trenty, Yumeng Zhang. Coupling of a two phase gas liquid compositional 3D Darcy flow with a 1D compositional free gas flow. The International Symposium of Finite Volumes for Complex Applications VII, Jun 2014, Berlin, Germany. 10.1007/978-3-319-055916_51. hal-01320500

\author{
HAL Id: hal-01320500 \\ https://hal.science/hal-01320500
}

Submitted on 24 May 2016

HAL is a multi-disciplinary open access archive for the deposit and dissemination of scientific research documents, whether they are published or not. The documents may come from teaching and research institutions in France or abroad, or from public or private research centers.
L'archive ouverte pluridisciplinaire HAL, est destinée au dépôt et à la diffusion de documents scientifiques de niveau recherche, publiés ou non, émanant des établissements d'enseignement et de recherche français ou étrangers, des laboratoires publics ou privés. 


\title{
Coupling of a two phase gas liquid compositional 3D Darcy flow with a 1D compositional free gas flow
}

\author{
K. Brenner, R. Masson, L. Trenty, Y. Zhang
}

\begin{abstract}
A model coupling a three dimensional gas liquid compositional Darcy flow and a one dimensional compositional free gas flow is presented. The coupling conditions at the interface between the gallery and the porous media account for the molar normal fluxes continuity for each component, the gas liquid thermodynamical equilibrium, the gas pressure continuity and the gas and liquid molar fractions continuity. This model is applied to the simulation of the mass exchanges at the interface between the repository and the ventilation excavated gallery in a nuclear waste geological repository. The convergence of the Vertex Approximate Gradient discretization is analysed for a simplified model coupling the Richards approximation in the porous media and the gas pressure equation in the gallery.
\end{abstract}

\section{Model}

Let $\omega$ and $S \subset \omega$ be two simply connected domains of $\mathbb{R}^{2}$ and $\Omega=(0, L) \times(\omega \backslash \bar{S})$ be the cylindrical domain defining the porous media. The excavated gallery corresponds to the domain $(0, L) \times S$ and it is assumed that the free flow in the gallery depends only on the $x$ coordinate along the gallery and on the time $t$. Let us denote

K. Brenner

University Nice Sophia Antipolis, and team COFFEE, INRIA Sophia Antipolis Méditerranée, France, e-mail: konstantin.brenner@unice.fr

R. Masson

University Nice Sophia Antipolis, and team COFFEE, INRIA Sophia Antipolis Méditerranée, France, e-mail: roland.masson@unice.fr

L. Trenty

Andra, 1 rue Jean Monnet, 92290 Chatenay-Malabry, France, e-mail: laurent.trenty@ andra.fr

Y. Zhang

University Nice Sophia Antipolis, and team COFFEE, INRIA Sophia Antipolis Méditerranée, France, e-mail: y.zhang56@yahoo.fr 
by $\Gamma=(0, L) \times \partial S$ the interface between the gallery and the porous media and by $\Gamma_{D}=((0, L) \times \partial \omega) \cup(\{0\} \times(\omega \backslash \bar{S})) \cup(\{L\} \times(\omega \backslash \bar{S}))$ the remaining boundaries of $\Omega$.

Let $\alpha=g, l$ denote the gas and liquid phases assumed to be both defined by a mixture of components $i \in \mathscr{C}$ among which the water component denoted by $e$ which can vaporize in the gas phase, and a set of gaseous components $j \in \mathscr{C} \backslash\{e\}$ which can dissolve in the liquid phase. For the sake of simplicity, the model will be assumed to be isothermal with a fixed temperature $T$. We will denote by $c^{\alpha}=$ $\left(c_{i}^{\alpha}, i \in \mathscr{C}\right)$ the vector of molar fractions of the components in the phase $\alpha=g, l$ with $\sum_{i \in \mathscr{C}} c_{i}^{\alpha}=1$, and by $P^{g}$ and $P^{l}$ the two phase pressures. The mass densities of the phases are denoted by $\rho^{\alpha}\left(P^{\alpha}, c^{\alpha}\right)$ and the molar densities by $\zeta^{\alpha}\left(P^{\alpha}, c^{\alpha}\right)$, $\alpha \in \mathscr{P}$. They are related by $\rho^{\alpha}\left(P^{\alpha}, c^{\alpha}\right)=\left(\sum_{i \in \mathscr{C}} c_{i}^{\alpha} M_{i}\right) \zeta^{\alpha}\left(P^{\alpha}, c^{\alpha}\right)$, where $M_{i}, i \in \mathscr{C}$ are the molar masses of the components. For the sake of simplicity, it is assumed that the liquid molar density $\zeta^{l}$ is constant as well as the viscosities $\mu^{\alpha}, \alpha=g, l$.

The two phase Darcy's laws are characterized by the relative permeability functions $k_{r}^{\alpha}\left(\mathbf{x}, S^{\alpha}\right)$, for both phases $\alpha=g, l$, and by the capillary pressure function $P_{c}\left(\mathbf{x}, S^{l}\right)$, where $S^{\alpha}, \alpha=l, g$ denote the saturations of the phases with $S^{g}+S^{l}=1$.

Each component $i \in \mathscr{C}$ is assumed to be at thermodynamical equilibrium between both phases which is characterized by the equality of its fugacities $f_{i}^{\alpha}, \alpha=g, l$ if both phases are present. The fugacities of the components in the gas phase are given by Dalton's law for an ideal mixture of perfect gas $f_{i}^{g}=c_{i}^{g} P^{g}, i \in \mathscr{C}$. The fugacities of the components in the liquid phase are given by Henry's law for the dissolution of the gaseous components in the liquid phase $f_{j}^{l}=c_{j}^{l} H_{j}(T), j \in$ $\mathscr{C} \backslash\{e\}$, and by Raoult-Kelvin's law for the water component in the liquid phase $f_{e}^{l}=c_{e}^{l} P_{s a t}(T) \exp \left(\frac{-\left(P^{g}-P^{l}\right)}{\zeta^{l} R T}\right)$, where $P_{s a t}(T)$ is the vapor pressure of the pure water.

Following [3], the gas liquid Darcy flow formulation uses both phase pressures $P^{g}$ and $P^{l}$ and the component fugacities $f=\left(f_{i}, i \in \mathscr{C}\right)$ as set of primary unknowns. For this set of unknowns, the component molar fractions of an absent phase are extended by those at equilibrium with the present phase leading to define $c_{i}^{\alpha}\left(f, P^{g}, P^{l}\right), \alpha=$ $g, l, i \in \mathscr{C}$ by

$$
\left\{\begin{array}{l}
c_{e}^{l}=\frac{f_{e}}{P_{\text {sat }}(T)} \exp \left(\frac{\left(P^{g}-P^{l}\right)}{\zeta^{l} R T}\right), \quad c_{j}^{l}=\frac{f_{j}}{H_{j}(T)}, j \in \mathscr{C} \backslash\{e\} \\
c_{e}^{g}=\frac{f_{e}}{P^{g}}, \quad c_{j}^{g}=\frac{f_{j}}{P^{g}}, j \in \mathscr{C} \backslash\{e\} .
\end{array}\right.
$$

The pressure of an absent phase is also extended by the buble (for gas) and by the dew (for liquid) pressure leading to the equations $\sum_{i \in \mathscr{C}} c_{i}^{\alpha}\left(f, P^{g}, P^{l}\right)=1, \alpha=$ $g, l$. Finally, we define $\mathscr{S}^{l}(\mathbf{x},$.$) as the inverse of the monotone graph extension of$ the opposite of the capillary pressure $-P_{c}(\mathbf{x},$.$) . This leads to the following set of$ equations in the porous media: 


$$
\left\{\begin{array}{l}
\phi \partial_{t} \sum_{\alpha \in \mathscr{P}} \zeta^{\alpha} S^{\alpha} c_{i}^{\alpha}+\operatorname{div}\left(\sum_{\alpha \in \mathscr{P}} \zeta^{\alpha} c_{i}^{\alpha} \mathbf{V}^{\alpha}\right)=0, i \in \mathscr{C}, \\
\mathbf{V}^{\alpha}=-\frac{k_{r}^{\alpha}\left(\mathbf{x}, S^{\alpha}\right)}{\mu^{\alpha}} \mathbf{K}\left(\nabla P^{\alpha}-\rho^{\alpha} \mathbf{g}\right), \alpha=g, l \\
S^{g}+S^{l}=1, \quad S^{l}=\mathscr{S}^{l}\left(\mathbf{x}, P^{l}-P^{g}\right), \quad \sum_{i \in \mathscr{C}} c_{i}^{\alpha}\left(f, P^{g}, P^{l}\right)=1, \alpha=g, l
\end{array}\right.
$$

In the gallery, the primary unknowns, depending only on the $x$ coordinate along the gallery and on the time $t$, are chosen to be the gas pressure $p$ and the gas molar fractions $c=\left(c_{i}, i \in \mathscr{C}\right)$. The set of equations is defined by the following no pressure wave isothermal pipe flow model where $\alpha>0, \beta>0$ are parameters for the pressure drop along the gallery, $\mathbf{n}$ is the unit normal vector at $\Gamma$ outward to $\Omega$, and $|S|$ is the surface of the section $S$.

$$
\left\{\begin{aligned}
\partial_{t}\left(|S| \zeta^{g}(p) c_{i}\right)+\partial_{x}\left(|S| \zeta^{g}(p) c_{i} w\right) & =\int_{\partial S} \sum_{\alpha=g, l} \zeta^{\alpha} c_{i}^{\alpha} \mathbf{V}^{\alpha} \cdot \mathbf{n} d s \\
\sum_{i \in \mathscr{C}} c_{i} & =1, \quad(\alpha w+\beta|w| w)=-\partial_{x} p
\end{aligned}\right.
$$

At the interface $\Gamma$ between the gallery and the porous media the coupling conditions are an adaptation to a 1D model in the gallery of [4]. Compared with [4], the gas pressure jump at the interface is neglected since a small flow rate between the porous media and the gallery is assumed due to the low permeability of the storage. Hence the coupling conditions account first for the continuity of the gas phase pressure $P^{g}=p$. Second, we impose the continuity of the gas molar fractions $c^{g}=c$, and third the thermodynamical equilibrium $f_{i}=f_{i}^{l}=f_{i}^{g}=p c_{i}$ for all $i \in \mathscr{C}$ together with $\sum_{i \in \mathscr{C}} c_{i}^{l}=1$ which provides the additional equation (using (1)):

$$
P^{g}-P^{l}=-\zeta^{l} R T \ln \left(\frac{f_{e}}{P_{s a t}(T)\left(1-\sum_{j \in \mathscr{C} \backslash\{e\}} \frac{f_{j}}{H_{j}(T)}\right)}\right) .
$$

\section{Numerical test}

Let $\omega$ and $S$ be the disks of center 0 and radius respectively $r_{\omega}=10 \mathrm{~m}$ and $r_{S}=2$ $\mathrm{m}$. We consider a radial mesh of the domain $(0, L) \times(\omega \backslash \bar{S}), L=100 \mathrm{~m}$, exponentially refined at the interface of the gallery $\Gamma$ to account for the steep gradient of the capillary pressure. In addition to the water component $e$, we consider the air gaseous component denoted by $a$ with the Henry constant $H_{a}=610^{9} \mathrm{~Pa}$. The gas molar density is given by $\zeta^{g}=\frac{p}{R T}$. The porous medium is initially saturated by the liquid phase with imposed pressure $P_{\text {init }}^{l}=4010^{5} \mathrm{~Pa}$ and composition $c_{a}^{l}=0, c_{e}^{l}=1$. At the external boundary $r=r_{\omega}$ the water pressure is fixed to $P_{0}^{l}=P_{\text {init }}^{l}$, with an input composition $c_{a}^{l}=0, c_{e}^{l}=1$. On both sides $x=0$ and $x=L$ of the porous media, zero flux boundary conditions are imposed. At the left side of the gallery $x=0$, we consider a given velocity $w=w_{0}$ and an input relative humidity $H_{r}=H_{r, 0}=0.5$ with $H_{r}=\frac{c_{e} p}{P_{s a t}(T)}$. The initial condition in the gallery 


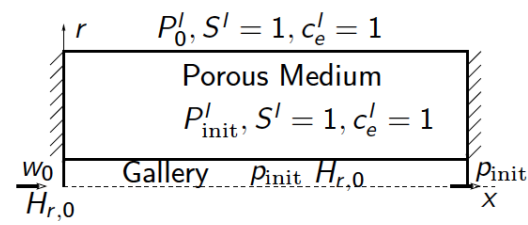

Fig. $1(x, r)$ cut of the storage and initial and boundary conditions of the test case.

is given by $p_{\text {init }}=10^{5} \mathrm{~Pa}$ and $H_{r}=H_{r, 0}$, and the pressure $p=p_{\text {init }}$ is fixed at the right side of the gallery (see Figure 1). The relative permeabilities and capillary pressure are given by the Van-Genuchten laws with the parameters $n=1.54$, $S_{r}^{l}=0.01, S_{r}^{g}=0, P_{r}=210^{6} \mathrm{~Pa}$ accounting for concrete rocktype with homogeneous isotropic permeability $K=10^{-18} \mathrm{~m}^{2}$ and porosity $\phi=0.3$. For the pressure load we have taken $\alpha=0$ and $\beta=10^{-3} \mathrm{~kg} \mathrm{~m}^{-4}$. The simulation is run over a period of 1500 days with an initial time step of 1 second and a maximum time step of 10 days. The input velocity $w_{0}$ is fixed to $w_{0}=1 \mathrm{~m} / \mathrm{s}$ during the first 400 days, $w_{0}=0.01 \mathrm{~m} / \mathrm{s}$ during the next 600 days, and $w_{0}=0 \mathrm{~m} / \mathrm{s}$ during the last 500 days. In order to validate the simulation, an approximate stationary solution is computed for each $w_{0}$ assuming that we can neglect the dissolution of air, the gravity, the pressure drop in the gallery, and that the longitudinal derivatives are small compared with the radial derivatives in the porous media. Then, the stationary solution $c_{e}(x), x \in(0, L)$ can be approximated by the solution of the following ODE for $w_{0}>0: \zeta^{g} w_{0}\left(1-c_{e, 0}\right) \partial_{x}\left(\frac{c_{e}(x)}{1-c_{e}(x)}\right)=\frac{2}{r_{S}^{2}} V_{T}\left(p_{c}\left(c_{e}(x)\right)\right), x \in(0, L)$ with $V_{T}\left(p_{c}\right)=$ $\frac{\zeta^{l} K}{\mu^{l} \log \left(\frac{r_{\omega}}{r_{S}}\right)}\left(P_{0}^{l}-p_{\text {init }}+\int_{0}^{p_{c}} k_{r}^{l}\left(\mathscr{S}^{l}(-u)\right) d u\right)$, and $p_{c}\left(c_{e}\right)=-\zeta^{l} R T \ln \left(\frac{p_{\text {init }} c_{e}}{P_{\text {sat }}(T)}\right)$, using the boundary condition $c_{e}(0)=c_{e, 0}=\frac{H_{r, 0} P_{\text {sat }}(T)}{p_{\text {init }}}$, and by $H_{r}(x)=\exp \left(\frac{P_{0}^{l}-p_{\text {init }}}{\zeta^{l} R T}\right)$, $x \in(0, L)$ for $w_{0}=0$. In Figure 2, we plot the average relative humidity in the gallery as well as the volume of gas in the porous medium function of time. Figure 3 plots the stationary numerical solution obtained for the gas saturation at the interface and in the porous media for each $w_{0}$. At the opening of the gallery at $t=0$, we observe in Figure 2 an increase of $H_{r}$ up to almost 1 in average in a few seconds due to a large liquid flow rate at the interface. Then, the flow rate decreases and we observe a drying of the gallery due to the ventilation at $w_{0}=1 \mathrm{~m} / \mathrm{s}$ down to an average relative humidity slightly above $H_{r, 0}$ in a few days. Meanwhile the gas penetrate slowly into the porous medium reaching a stationnary state with around $13.5 \mathrm{~m}^{3}$ of gas in say 400 days. When the input velocity is reduced to $0.01 \mathrm{~m} / \mathrm{s}$, we observe first a rapid increase of $H_{r}$ in say 1 day due to the reduced ventilation followed by a convergence to a second stationnary state with $H_{r}=0.77$ in average in the gallery and $12 \mathrm{~m}^{3}$ of gas in the porous medium. When $w_{0}$ is set to $0, H_{r}$ reaches a value above 1 corresponding to $S^{l}=1$ at the interface and the gas disappears from the porous medium in around 100 days.

Figure 2 also compares the stationary numerical relative humidity obtained for each $w_{0}$ with its approximate "analytical" solution. A very good match is obtained. 

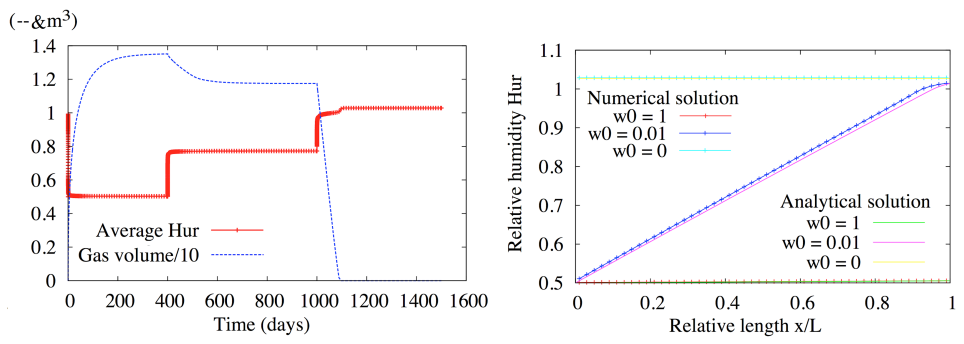

Fig. 2 Average of the relative humidity in the gallery and volume of gas in the porous medium function of time (left); stationary relative humidity for each $w_{0}$ compared with its approximate "analytical" solution (right).

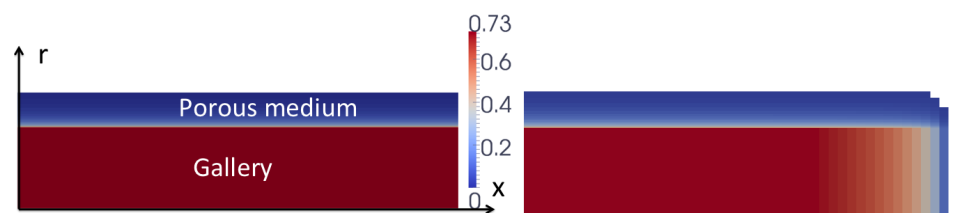

Fig. $3(x, r)$ cut of the stationary gas saturation at the interface (depending only on $x$ for $r \in\left(0, r_{S}\right)$ ) and in the porous medium (for $r>r_{S}$ ) for $w_{0}=1 \mathrm{~m} / \mathrm{s}$ (left), and $w_{0}=0.01 \mathrm{~m} / \mathrm{s}$ (right). Only the values above the threshold $10^{-3}$ are plotted.

\section{Convergence analysis of a simplified model}

We consider the following simplified model using the Richards approximation in the porous medium and a single component equation in the gallery with linear pressure drop

$$
\left\{\begin{array}{l}
\phi \partial_{t}\left(\zeta^{l} \mathscr{S}^{l}(., u)\right)+\operatorname{div}\left(\zeta^{l} \mathbf{V}^{l}\right)=0 \\
\partial_{t}\left(|S| \zeta^{g}(p)\right)+\partial_{x}\left(-\frac{1}{\alpha}|S| \tilde{\zeta}^{g}(p) \partial_{x} p\right)=\int_{\partial S} \zeta^{l} \mathbf{V}^{l} \cdot \mathbf{n} d s \\
\mathbf{V}^{l}=-\frac{k_{r}^{\alpha}\left(., \mathscr{S}^{l}(., u)\right)}{\mu^{l}} \mathbf{K}\left(\nabla u-M^{l} \zeta^{l} \mathbf{g}\right), p=g(\gamma(u))
\end{array}\right.
$$

where $\gamma$ denotes the trace operator from $H^{1}(\Omega)$ to $H^{1 / 2}(\Gamma)$. The only primary unknown in the porous media is the liquid pressure denoted by $u$. The liquid mass density is assumed to be fixed to $M^{l} \zeta^{l}$ where $M^{l}$ is the molar mass of the liquid phase. The thermodynamical equilibrium at the interface $\Gamma$ is accounted for by the relation $p=g(\gamma(u))$ with $g \in C^{1}(\mathbb{R}, \mathbb{R}), 0<c_{1} \leq g^{\prime}(q) \leq c_{2}$ for all $q \in \mathbb{R}$ and for given constants $c_{1}, c_{2}$. The function $g$ is a regularization for large positive and negative $u$ of $p=\frac{P_{s a t}}{c_{e}} e^{\frac{u}{\zeta^{l} R T}}$ for given constants $1 \geq c_{e}>0$ and $T>0$. The molar gas density is set to $\zeta^{g}(p)=\frac{p}{R T}$ and is truncated in the flux term such that $\tilde{\zeta}^{g}(p)$ is assumed to be a non decreasing function in $C^{1}(\mathbb{R}, \mathbb{R})$ bounded from below and above by two strictly positive constants and with a bounded derivative. 
Let $\gamma_{e}$ be the trace operator from $H^{1}(\Omega)$ to $H^{1 / 2}\left(\Gamma_{D}\right)$. We define the function space $V=\left\{u \in H^{1}(\Omega) \mid \gamma u \in H^{1}(\Gamma), \partial_{s} \gamma u=0\right\}$, where $s$ denotes the curvilinear coordinate along $\partial S$. Taking into account homogeneous Dirichlet boundary conditions, its subspace is denoted by $V^{0}=\left\{u \in V \mid \gamma_{e} u=0,(\gamma u)(0)=(\gamma u)(L)=0\right\}$, endowed with the norm $\|u\|_{V^{0}}^{2}=\int_{\Omega}|\nabla u(\mathbf{x})|^{2} d \mathbf{x}+\int_{0}^{L}\left|\frac{d}{d x} \gamma u(x)\right|^{2} d x$.

Let $\mathscr{C}\left(\Omega \times\left[0, T_{f}\right)\right)$ be the subspace of functions $\varphi$ of $C^{\infty}\left(\bar{\Omega} \times\left[0, T_{f}\right]\right)$ vanishing at $t=T_{f}$ and at $\Gamma_{D}$ and such that $\partial_{s} \varphi=0$ on $(0, L) \times \partial S$. Given $\bar{u} \in V$ and $u_{\text {ini }} \in V$, the variational formulation of the simplified coupled model amounts to find $u$ with $u-\bar{u} \in L^{2}\left(0, T_{f} ; V^{0}\right)$ such that for all $\varphi \in \mathscr{C}\left(\Omega \times\left[0, T_{f}\right)\right)$ one has

$$
\left\{\begin{array}{l}
-\int_{0}^{T_{f}} \int_{\Omega} \phi(\mathbf{x}) \zeta^{l} \mathscr{S}^{l}(\mathbf{x}, u(\mathbf{x}, t)) \partial_{t} \varphi(\mathbf{x}, t) d \mathbf{x} d t-\int_{\Omega} \phi \zeta^{l} \mathscr{S}^{l}\left(\mathbf{x}, u_{i n i}(\mathbf{x})\right) \varphi(\mathbf{x}, 0) d \mathbf{x} \\
-\int_{0}^{T_{f}} \int_{0}^{L}|S| \zeta^{g}(g(\gamma u)(x, t)) \partial_{t} \gamma \varphi(x, t) d x d t-\int_{0}^{L}|S| \zeta^{g}\left(g\left(\gamma\left(u_{i n i}\right)\right)(x)\right) \gamma \varphi(x, 0) d x \\
+\int_{0}^{T_{f}} \int_{\Omega} \zeta^{l} \frac{k_{r}^{l}\left(\mathbf{x}, \mathscr{S}^{l}(u(\mathbf{x}, t))\right)}{\mu^{l}} \mathbf{K}\left(\nabla u(\mathbf{x}, t)-M^{l} \zeta^{l} \mathbf{g}\right) \cdot \nabla \varphi(\mathbf{x}, t) d \mathbf{x} d t \\
+\int_{0}^{T_{f}} \int_{0}^{L} \frac{1}{\alpha(x)}|S| \widetilde{\zeta}^{g}(g(\gamma u)(x, t)) \partial_{x} g(\gamma u)(x, t) \partial_{x} \gamma \varphi(x, t) d x d t=0 .
\end{array}\right.
$$

We make the following additional assumptions on the data:

- It is assumed that $k_{r}^{l}(\mathbf{x}, s)$ is a measurable function w.r.t. $\mathbf{x}$ and continuous w.r.t. $s$, and such that $0<k_{\min } \leq k_{r}^{l}(\mathbf{x}, s) \leq k_{\max }$ for all $(\mathbf{x}, s) \in \Omega \times[0,1]$.

- $\mathscr{S}^{l}(\mathbf{x}, u) \in[0,1]$ for all $(\mathbf{x}, u) \in \Omega \times \mathbb{R}$ with $\mathscr{S}^{l}(\mathbf{x}, u)=\mathscr{S}_{j}^{l}(u)$ for a.e. $\mathbf{x} \in \Omega_{j}$ and all $u \in \mathbb{R}$, where $\mathscr{S}_{j}^{l}$ is a non decreasing Lipschitz continuous function with constant $L_{S}$ and $\left(\Omega_{j}\right)_{j \in J}$ is a finite family of disjoint connected polyhedral open sets such that $\bigcup_{j \in J} \overline{\Omega_{j}}=\bar{\Omega}$.

- The permeability tensor $\mathbf{K}$ is a measurable function on the space of symmetric 3 dimensional matrices such that there exist $0<\lambda_{\min } \leq \lambda_{\max }$ with $\lambda_{\min }|\xi|^{2} \leq$ $(\mathbf{K}(\mathbf{x}) \xi, \xi) \leq \lambda_{\max }|\xi|^{2}$ for all $\mathbf{x} \in \bar{\Omega}$.

- $\alpha \in L^{\infty}(0, L)$ is such that $0<\alpha_{\text {min }} \leq \alpha(x) \leq \alpha_{\max }$ for all $x \in(0, L)$.

- The porosity $\phi$ belongs to $L^{\infty}(\Omega)$ with $0<\phi_{\min } \leq \phi(\mathbf{x}) \leq \phi_{\max }$ for all $\mathbf{x} \in \Omega$.

Vertex Approximate Gradient (VAG) discretization: We assume that $\omega$ and $S$ are polygonal domains of $\mathbb{R}^{2}$ and we consider a conforming polyhedral mesh of the domain $\Omega$. It is assumed that the intersection of the mesh with the boundary $\Gamma$ of the gallery is the tensor product of the $1 \mathrm{D}$ mesh of $(0, L)$ defined by $0=x_{0}<$ $x_{1}<\cdots<x_{n_{x}+1}=L$ by the 1D mesh of $\partial S$ defined by the set of distinct points $\mathbf{s}_{1}, \mathbf{s}_{2} \cdots, \mathbf{s}_{n_{S}}, \mathbf{s}_{n_{S}+1}=\mathbf{s}_{1}$ of $\partial S$ in cyclic order.

Let $\mathscr{M}$ denote the set of cells $K, \mathscr{V}$ the set of nodes $\mathbf{s}, \mathscr{E}$ the set of edges $e$, and $\mathscr{F}$ the set of faces $\sigma$, of the mesh. We denote by $\mathscr{V}_{K}$ the set of nodes of the cell $K \in \mathscr{M}$, by $\mathscr{V}_{\sigma}$ the set of nodes and by $\mathscr{E}_{\sigma}$ the set of edges of the face $\sigma \in \mathscr{F}$. The set of nodes of the mesh belonging to $\left\{x_{i}\right\} \times \partial S$ is denoted by $\mathscr{V}_{i}$ for all $i=0, \cdots, n_{x}+1$.

It is assumed for each face $\sigma \in \mathscr{F}$, that there exists a so-called "centre" of the face $\mathbf{x}_{\sigma}$ such that $\mathbf{x}_{\sigma}=\sum_{\mathbf{s} \in \mathscr{V}_{\sigma}} \beta_{\sigma, \mathbf{s}} \mathbf{x}_{\mathbf{s}}$, with $\sum_{\mathbf{s} \in \mathscr{V}_{\sigma}} \beta_{\sigma, \mathbf{s}}=1$, where $\beta_{\sigma, \mathbf{s}} \geq 0$ for all 
$\mathbf{s} \in \mathscr{V}_{\sigma}$. The face $\sigma$ is assumed to be star-shaped w.r.t. its centre $\mathbf{x}_{\sigma}$ which means that the face $\sigma$ matches with the union of the triangles $\tau_{\sigma, e}$ defined by the face centre $\mathbf{x}_{\sigma}$ and each of its edge $e \in \mathscr{E}_{\sigma}$.

The previous discretization is denoted by $\mathscr{D}$, and we define the discrete space

$$
\begin{aligned}
X_{\mathscr{D}}=\left\{v_{K} \in \mathbb{R}, v_{\mathbf{s}} \in \mathbb{R}, v_{i} \in \mathbb{R}, K \in \mathscr{M}, \mathbf{s} \in \mathscr{V}, i=0, \cdots, n_{x}+1\right. \\
\left.\mid v_{\mathbf{s}}=v_{i} \text { for all } \mathbf{s} \in \mathscr{V}_{i}, i=0, \cdots, n_{x}+1\right\},
\end{aligned}
$$

and its subspace with homogeneous Dirichlet boundary conditions on $\Gamma_{D}$ and at $x=0, x=L$

$$
X_{\mathscr{D}}^{0}=\left\{v \in X_{\mathscr{D}} \mid v_{\mathbf{s}}=0 \text { for all } \mathbf{s} \in \mathscr{V}_{D}\right\},
$$

where $\mathscr{V}_{D}=\mathscr{V} \cap \bar{\Gamma}_{D}$ are the Dirichlet boundary nodes.

Following [1], the extension of the VAG discretization to the coupled model (6) is based on conforming Finite Element reconstructions of the gradient operators on $\Omega$ and on $(0, L)$, and on non conforming piecewise constant function reconstructions on $\Omega$ and on $(0, L)$.

For all $\sigma \in \mathscr{F}$, let us first define the operator $I_{\sigma}: X_{\mathscr{D}} \rightarrow \mathbb{R}$ such that $I_{\sigma}(v)=$ $\sum_{\mathbf{s} \in \mathscr{V}_{\sigma}} \beta_{\sigma, \mathbf{s}} \mathbf{v}_{\mathbf{s}}$, which is by definition of $\mathbf{x}_{\sigma}$ a second order interpolation operator at point $\mathbf{x}_{\sigma}$.

Let us introduce the tetrahedral sub-mesh $\mathscr{T}=\left\{\tau_{K, \sigma, e}, e \in \mathscr{E}_{\sigma}, \sigma \in \mathscr{F}_{K}, K \in \mathscr{M}\right\}$ of the mesh $\mathscr{M}$, where $\tau_{K, \sigma, e}$ is the tetrahedron defined by the cell center $\mathbf{x}_{K}$ and the triangle $\tau_{\sigma, e}$. For a given $v \in X_{\mathscr{D}}$, we define the function $\Pi_{\mathscr{T}} v \in V$ as the continuous piecewise affine function on each tetrahedron $\tau$ of $\mathscr{T}$ such that $\Pi_{\mathscr{T}} v\left(\mathbf{x}_{K}\right)=v_{K}$, $\Pi_{\mathscr{T}} v(\mathbf{s})=v_{\mathbf{s}}$, and $\Pi_{\mathscr{T}} v\left(\mathbf{x}_{\sigma}\right)=I_{\sigma}(v)$ for all $K \in \mathscr{M}, \mathbf{s} \in \mathscr{V}, \sigma \in \mathscr{F}$.

It is easily checked that $\partial_{s} \gamma \Pi_{\mathscr{T}} v=0$ which shows that $\Pi_{\mathscr{T}} v \in V$ for all $v \in X_{\mathscr{D}}$. Then, the gradient operators are defined for all $v \in X_{\mathscr{D}}$ by

$$
\nabla_{\mathscr{D} v}=\nabla \Pi_{\mathscr{T}} v \text { and } \nabla_{x, \mathscr{D}} v=\partial_{x} \gamma \Pi_{\mathscr{T}} v
$$

One can easily check that $\nabla_{x, D} v=\frac{v_{i+1}-v_{i}}{x_{i+1}-x_{i}}$ on $\left(x_{i}, x_{i+1}\right)$ for all $i=0, \cdots, n_{x}$. For the reconstructions of functions operators, we first set

$$
\Pi_{\mathscr{D}} v(\mathbf{x})=v_{K} \text { for all } \mathbf{x} \in K, K \in \mathscr{M} .
$$
Next, let us define the points $x_{i+\frac{1}{2}}=\frac{x_{i}+x_{i+1}}{2}, i=1, \cdots, n_{x}-1, x_{\frac{1}{2}}=0, x_{n_{x}+\frac{1}{2}}=L$,
we set

$$
\Pi_{x, \mathscr{D} v}(x)=v_{i} \text { for all } x \in\left(x_{i-\frac{1}{2}}, x_{i+\frac{1}{2}}\right), i=1, \cdots, n_{x} .
$$

Let $\rho_{\tau}$ denote the insphere diameter of a given tetrahedron $\tau \in \mathscr{T}, h_{\tau}$ its diameter, $h_{\mathscr{T}}=\max _{\tau \in \mathscr{T}} h_{\tau}$, and $\theta_{\mathscr{T}}=\max _{\tau \in \mathscr{T}} \frac{h_{\tau}}{\rho_{\tau}}$ and $\gamma_{\mathscr{M}}=\max _{K \in \mathscr{M}} \operatorname{Card}\left(\mathscr{V}_{K}\right)$. For $N \in \mathbb{N}^{*}$, let us consider the time discretization $t^{0}=0<t^{1}<\cdots<t^{n-1}<t^{n} \cdots<$ $t^{N}=T_{f}$ of the time interval $\left[0, T_{f}\right]$. We denote the time steps by $\Delta t^{n}=t^{n}-t^{n-1}$ for all $n=1, \cdots, N$. For $v \in X_{\mathscr{D}}$, and a function $k \in C^{0}(\mathbb{R}, \mathbb{R})$, we define $k(v) \in X_{\mathscr{D}}$ as follows: $k(v)_{\mathbf{s}}=k\left(v_{\mathbf{s}}\right)$ for all $\mathbf{s} \in \mathscr{V}, k(v)_{K}=k\left(v_{K}\right)$ for all $K \in \mathscr{M}$, and $k(v)_{i}=k\left(v_{i}\right)$ for all $i=0, \cdots, n_{x}+1$. 
Given $u_{\mathscr{D}}^{0} \in X_{\mathscr{D}}$ and $\bar{u}_{\mathscr{D}} \in X_{\mathscr{D}}$, the discretization of the coupled model (6) looks for $u_{\mathscr{D}}^{n} \in X_{\mathscr{D}}$ with $u_{\mathscr{D}}^{n}-\bar{u}_{\mathscr{D}} \in X_{\mathscr{D}}^{0}$ for all $n=1, \cdots, N$ such that for all $v_{\mathscr{D}} \in X_{\mathscr{D}}^{0}$

$$
\left\{\begin{array}{l}
\int_{\Omega} \phi(\mathbf{x}) \zeta^{l} \frac{\mathscr{S}^{l}\left(\mathbf{x}, \Pi_{\mathscr{D}} u_{\mathscr{D}}^{n}(\mathbf{x})\right)-\mathscr{S}^{l}\left(\mathbf{x}, \Pi_{\mathscr{D}} u_{\mathscr{D}}^{n-1}(\mathbf{x})\right)}{\Delta t^{n}} \Pi_{\mathscr{D} v_{\mathscr{D}}}(\mathbf{x}) d \mathbf{x} \\
+\int_{0}^{L}|S| \frac{\zeta^{g}\left(\Pi_{x, \mathscr{D}} g\left(u_{\mathscr{D}}^{n}\right)(x)\right)-\zeta^{g}\left(\Pi_{x, \mathscr{D}} g\left(u_{\mathscr{D}}^{n-1}\right)(x)\right)}{\Delta t^{n}} \Pi_{x, \mathscr{D}} v_{\mathscr{D}}(x) d x \\
+\int_{\Omega} \zeta^{l} \frac{k_{r}^{l}\left(\mathbf{x}, \mathscr{S}^{l}\left(\mathbf{x}, \Pi_{\mathscr{D}} u_{\mathscr{D}}^{n}(\mathbf{x})\right)\right)}{\mu^{l}} \mathbf{K}\left(\nabla_{\mathscr{D}} u_{\mathscr{D}}^{n}(\mathbf{x})-M^{l} \zeta^{l} \mathbf{g}\right) \cdot \nabla_{\mathscr{D}} v_{\mathscr{D}}(\mathbf{x}) d \mathbf{x} \\
+\int_{0}^{L} \frac{1}{\alpha(x)}|S| \widetilde{\zeta}^{g}\left(\Pi_{x, \mathscr{D}} g\left(u_{\mathscr{D}}^{n}\right)(x)\right) \nabla_{x, \mathscr{D}} g\left(u_{\mathscr{D}}^{n}\right)(x) \nabla_{x, \mathscr{D}} v_{\mathscr{D}}(x) d x=0 .
\end{array}\right.
$$

Convergence analysis: Let us set $X_{\mathscr{D}, \Delta t}=\left(X_{\mathscr{D}}\right)^{N}$, and for all $v_{\mathscr{D}}=\left(v_{\mathscr{D}}^{n}\right)_{n=1, \cdots, N} \in$ $X_{\mathscr{D}, \Delta t}$ let us define for all $n=1, \cdots, N$, and for all $(\mathbf{x}, t) \in \Omega \times\left(t^{n-1}, t^{n}\right]$ the functions $\Pi_{\mathscr{D}, \Delta t} v_{\mathscr{D}}(\mathbf{x}, t)=\Pi_{\mathscr{D}} v_{\mathscr{D}}^{n}(\mathbf{x}), \Pi_{x, \mathscr{D}, \Delta t} v_{\mathscr{D}}(x, t)=\Pi_{x, \mathscr{D}} v_{\mathscr{D}}^{n}(x), \Pi_{\mathscr{T}, \Delta t} v_{\mathscr{D}}(\mathbf{x}, t)=$ $\Pi_{\mathscr{T}} v_{\mathscr{D}}^{n}(\mathbf{x})$. Let $u_{\mathscr{D}}=\left(u_{\mathscr{D}}^{n}\right)_{n=1, \cdots, N}$, the given solution to (8), we also define the functions $S_{\mathscr{D}, \Delta t}^{l}(\mathbf{x}, t)=\mathscr{S}^{l}\left(\mathbf{x}, \Pi_{\mathscr{D}, \Delta t} u_{\mathscr{D}}(\mathbf{x}, t)\right), p_{x, \mathscr{D}, \Delta t}(x, t)=g\left(\Pi_{x, \mathscr{D}, \Delta t} u_{\mathscr{D}}(x, t)\right)$. Using similar techniques as in [2], we can prove the following convergence theorem.

Theorem 1. Let $\mathscr{D}^{(m)}, \Delta t^{n,(m)}, n=1, \cdots, N^{(m)}, m \in \mathbb{N}$ be a sequence of space time discretizations such that there exist $\theta>0, \gamma>0$ with $\theta_{\mathscr{T}^{(m)}} \leq \theta, \gamma_{\mathscr{T}^{(m)}} \leq \gamma$. It is assumed that $\lim _{m \rightarrow+\infty} h_{\mathscr{T}^{(m)}}=0$, and that $\Delta t^{(m)}=\max _{n=1, \cdots, N^{(m)}} \Delta t^{n,(m)}$ tends to zero when $m \rightarrow+\infty$, and that $\left\|\Pi_{\mathscr{D}^{(m)}} u_{\mathscr{D}^{(m)}}^{0}-u_{\text {ini }}\right\|_{L^{2}(\Omega)},\left\|\Pi_{x, \mathscr{D}(m)} u_{\mathscr{D}(m)}^{0}-\gamma u_{\text {ini }}\right\|_{L^{2}(0, L)}$, $\left\|\Pi_{\mathscr{T}^{(m)}} \bar{u}_{\mathscr{D}^{(m)}}-\bar{u}\right\|_{V^{0}},\left\|\Pi_{\mathscr{D}^{(m)}} \bar{u}_{\mathscr{D}^{(m)}}-\bar{u}\right\|_{L^{2}(\Omega)},\left\|\Pi_{x, \mathscr{D}^{(m)}} \bar{u}_{\mathscr{D}^{(m)}}-\gamma \bar{u}\right\|_{L^{2}(0, L)}$ tends to zero when $m \rightarrow+\infty$. Then, there exist a subsequence of $m \in \mathbb{N}$ and a function $u \in L^{2}\left(0, T_{f} ; V\right)$ solution of (6) such that up to this subsequence $S_{\mathscr{D}(m), \Delta t^{(m)}}^{l} \rightarrow$ $\mathscr{S}^{l}(., u)$ strongly in $L^{2}\left(\Omega \times\left(0, T_{f}\right)\right), \Pi_{\mathscr{D}(m)}, \Delta t^{(m)} u_{\mathscr{D}(m)} \rightarrow$ u weakly in $L^{2}\left(\Omega \times\left(0, T_{f}\right)\right)$, and $p_{x, \mathscr{D}}(m), \Delta t^{(m)} \rightarrow g(\gamma(u))$ strongly in $L^{2}\left((0, L) \times\left(0, T_{f}\right)\right)$.

Acknowledgements The authors would like to thank Andra for its financial support.

\section{References}

1. Eymard, R., Guichard, C., Herbin, R.: Small-stencil 3d schemes for diffusive flows in porous media. ESAIM: Mathematical Modelling and Numerical Analysis 46, 265-290 (2010)

2. Eymard, R., Guichard, C., Herbin, R., Masson, R.: Gradient schemes for two-phase flow in heterogeneous porous media and Richards equation. ZAMM - Journal of Applied Mathematics and Mechanics (2013). DOI 10.1002/zamm.201200206

3. Masson, R., Trenty, L., Zhang, Y.: Formulations of two-phase liquid gas compositional Darcy flows with phase transitions (2013). URL http://hal.archives-ouvertes.fr/hal-00910366

4. Mosthaf, K., Baber, K., Flemisch, B., Helmig, R., Leijnse, A., Rybak, I., Wohlmuth, B.: A coupling concept for two-phase compositional porous-medium and single-phase compositional free flow. Water Resources Research 47(10) (2011) 